\title{
Effects of surfactant's molecular weight on the wettability of vinyl polysiloxane impression materials after immersion disinfection
}

\author{
Shahab Ud Din1,7, Muhammad Hassan2,7, Sadia Khalid3, Muhammad Sohail Zafar4,5,*, \\ Bilal Ahmed6, Mangala Patel7
}

IDentistry and Allied Disciplines, Shaheed Zulfiqar Ali Bhutto Medical University (SZABMU), Pakistan Institute of Medical Sciences (PIMS), Islamabad 44000, Pakistan

${ }_{2}$ Department of Dental Materials, University College of Dentistry, UoL, Lahore 54600, Pakistan

3Dentistry and Allied Disciplines, Shaheed Zulfiqar Ali Bhutto Medical University (SZABMU), Pakistan Institute of Medical Sciences (PIMS), Islamabad 44000, Pakistan

4Department of Restorative Dentistry, College of Dentistry, Taibah University, Madina Munawwarrah 41311, Saudi Arabia

${ }_{5}$ Department of Dental Materials, Islamic International Dental College, Riphah International University, Islamabad 44000, Pakistan

${ }_{6}$ Department of Prosthodontics,National University of Sciences and Technology (NUST), Islamabad 44000, Pakistan

${ }_{7}$ Department of Oral Growth and Development (Dental Physical Sciences Unit), Bart's and The London School of Medicine and Dentistry, Queen Mary, University of London, London E1 4NS, $U K$

*Author to whom correspondence should be addressed.

Email: MZAFAR@ taibahu.edu.sa

\begin{abstract}
The aim of this study is to develop novel hydrophilic vinyl polysiloxane (VPS) materials incorporating novel hydrophilic agents (Rhodasurf CET-2 and CET-5). In addition, experimental material's wetting properties (contact angles) were compared with commercial materials upon immersion in various media. Three commercial (Aquasil UltraMonophase [Aq M], Elite HD-Monophase [Elt M], Extrude Medium-bodied [Extr M]) and five experimental (Exp I-V) materials were used in this study. The contact angles were measured at time intervals (10, 30, 60 and 120 seconds), immediately after setting and after
\end{abstract}


immersion using a Drop Shape Analysis (DSA). The results were analyzed statistically using the SPSS software.The largest contact angle (at $10 \mathrm{sec}$ ) was recorded for Elt M $(108.78 \pm 6.04)$ that was significantly larger $(\mathrm{p}<0.05)$ than Aq M $(36.19 \pm 4.05)$ and Extr $\mathrm{M}$

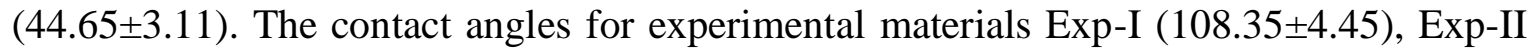
(104.08 \pm 4.70$)$ closely matched to Elt M $(108.78 \pm 6.04)$ however were significantly larger

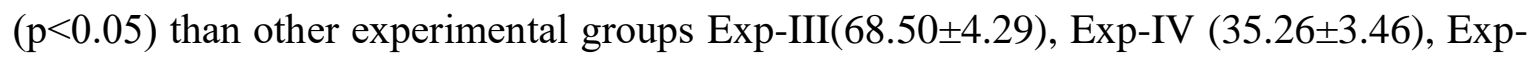

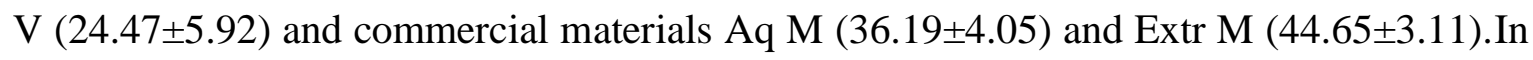
conclusion, the experimental materials containing the surfactant showed comparatively lower contact angles than commercially available VPS materials. The smaller molecular weight surfactant (Rhodasurf CET-2) resulted in lowering of contact angles compared to the larger molecular weight surfactant (Rhodasurf CET-5).

\section{Keywords:}

Dental biomaterials, Polymericimpression materials,Hydrophilic vinyl polysiloxane,Surfactant,Disinfection, contact angles.

\section{INTRODUCTION}

Polymeric materials are used for a variety of prosthodontic applications ranging from recording impression to fabricating dentures. $(1,2)$ Commonly used dental polymers include poly-methyl methacrylate(3-5), polyetheretherketone(6-8) and polymeric rubber base impression materials(9-11) such as vinyl polysiloxane (VPS). Among polymeric impression materials, the VPS impression materials are most commonly used due to their excellent elastic recovery and dimensional stability.(12,13) Therefore, VPS is capable of recording precise tissue details required for the construction of fine dental restorations such as inlays, crowns and bridges. $(9,14,15)$ However, a significant limitation of these materials is their inherent hydrophobicity $(9,16)$ and need of a dry field(16-18) to record the details. In order to 
overcome the problem of hydrophobicity, manufacturers have incorporated surfactants and developed hydrophilic VPS impression materials.(16, 18, 19) A number of studies have evaluated the hydrophilicity/wettability of impression materials after the material sets(18, 20, 21); however, the hydrophilicity of impression materials changes during the setting period.(22) Therefore, it is important to evaluate any changes occurring while materialis setting.

Many researchers $(9,23,24)$ have investigated various commercial materials and reported variation in properties (such as wettability, viscosity and compatibility with gypsum) corresponding to their compositions. Oh et al.,(20) developed a tailored compositions of VPS impression materials, in order to investigate the effect of surfactant onthe surface hydrophilicity of these materials on the basis of contact angles. The incorporation of surfactants reduced the contact angles of modified VPS impression materials.(20) In addition, a strong negative correlation has been reported between the surfactant's concentration and contact angle; the higher amount of surfactant resulted in lowering of the contact angles.(21, 25)

In addition, the disinfection of these materials was not performed that might have affected the materials properties. Dental impressions are always contaminated with saliva and most often there is blood on them. Blood and saliva can cause cross-contamination to the dental team.(22, 26-28) For infection control measures, impression materials are treated using disinfectants before proceeding to laboratory procedures. Therefore, to approximate the clinical protocol, impression materials should be disinfected prior to assess the hydrophilicity and wettability.(29) Different studies showed that the elastomeric impression materials including hydrophilic VPS impression materials can be disinfected for a time period ranging from $30 \mathrm{~min}(30), 1$ hour and18 hours.(31)However, the effects of long term disinfection on VPS impression materials have not been fully investigated. The aim of the current study was to develop novel hydrophilic vinyl polysiloxane (VPS) impression materials incorporating novel hydrophilic agents (Rhodasurf CET-2 and CET-5). In addition, the effects of 
surfactant's molecular weight on the hydrophilicity and wetting properties (contact angles) were compared with the controls and commercial materials upon immersion in various media.

\section{EXPERIMENTAL DETAILS}

\subsection{Materials}

Three commercially available medium-body vinyl polysiloxane (VPS) impression materials; Aquasil Ultra Monophase (Aq M) (Dentsply, USA), Elite HD Monophase (Elt M) (Zhermack, Italy), Extrude, (Extr M) (Kerr, USA) were used in this study. Based on results of pilot studies, the most favourable five VPS experimental formulations (Exp-I, Exp-II, ExpIII, Exp-IV and Exp-V) were prepared using various combinations of reagents (Table I). The proportion of base and catalyst paste was maintained at 50 weight $\%$ for each experimental formulation.For disinfection, $1 \%$ sodium hypochlorite $(\mathrm{NaOCl})$ prepared by the dilution of stock solution (Fisher Scientific, UK) was used. Deionized distilled water was used for the dilution of $\mathrm{NaOCl}$ stock solution for the immersion of all VPS impression materials. In case of experimental materials, Exp-I containing only basic components was used as a control for Exp-II that contained a novel cross-linking agent (TFDMSOS) in addition to basic components (Table 1).At the same time, Exp-II was used as a control for Exp-III, IV and V that incorporated the novel non-ionic surfactant (Rhodasurf CET-2) in variable concentrations in addition to basic components of Exp-II.

In order to evaluate the effects of surfactant's molecular weight, another experimental formulation (Exp-VI) was prepared. The composition of Exp-VI was exactly the same as described for Exp-III in Table I. The only exception was that the 2 wt. $\%$ of Rhodasurf CET2 in Exp-III was replaced by 2 wt. \% of higher molecular weight Rhodasurf CET-5 (Rhodia, UK) in Exp-VI. 
Table I. Formulations of novel experimental VPS (Exp-I, II, III, IV and V) polymer materials use in this study

$$
\text { Base paste (Weight \%) Catalyst paste (Weight }
$$

Components

$\%)$

\begin{tabular}{lccccccc}
\cline { 2 - 7 } & Exp & Exp- & Exp- & Exp- & \multicolumn{2}{c}{ Exp- } & \multicolumn{2}{c}{ Exp-I \& } & \multicolumn{2}{c}{ Exp-III, IV } \\
& -I & II & III & IV & V & II & \& V \\
\hline Vinyl-terminated & 39.9 & 39.9 & 37.95 & 37.46 & 36.98 & 40.72 & 39.51 \\
poly(dimethylsiloxane), & 0 & 0 & & & & & \\
Mw 62700 Da(Fluorochem, & & & & & & &
\end{tabular}

UK)

$\begin{array}{llllll}\text { Poly(methylhydrosiloxane), con } & 1.10 & 0.77 & 0.74 & 0.73 & 0.72\end{array}$

ventional cross-linking agent,

Mw 2270 Sigma-Aldrich,

(Dorset, UK)

Tetra-functional

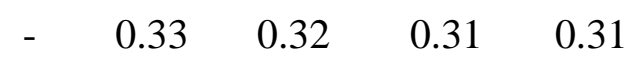

(dimethylsilyl) orthosilicate

(TFDMSOS), novel cross-

linking agent, $\mathrm{Mw} \sim 328.73$,

Sigma-Aldrich, (Dorset, UK)

Platinum catalyst $(0.05$

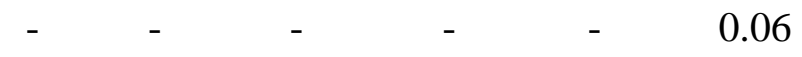

M)Sigma-Aldrich, (Dorset,

UK)

Rhodasurf CET-2

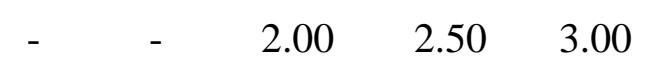

(Ethoxylatedcetyl-oleyl alcohol), surfactant, (Rhodia,

UK)

Palladium as scavenger

$-\quad-$

$(<1 \mu \mathrm{m})$, Sigma-Aldrich,

(Dorset, UK)

Aerosil R 812 S, fillers,

$\begin{array}{lll}9.00 & 9.00 & 9.00\end{array}$

9.00

$9.00 \quad 9.00$

9.00

(Lawrence Industries, UK)

Total Weight

$50 \%$

$\mathbf{5 0 \%}$

\subsection{Sample Preparation}

For contact angle measurements, rectangular shaped(40 x10 x2 mm) specimens were $\operatorname{prepared}(\mathrm{n}=10$ for each material) using stainless steel metal moulds as described 
previously.(21) Briefly, the stainless steel mould with acetate sheet top and bottom covers were positioned ona metal plate (Figure 1). The base and catalyst component of each material were mixed, using an auto-mixing syringe and extruded directly into the mould cavity with a slight overfilling. A homogenous pressure was applied to whole assembly unitusing a handoperated hydraulic press (MESTRA MOD-030350, TalleresMestraitua, Spain).In order to distribute the material evenly in the mould cavity,flushing out excess material and expel air bubbles, the pressure was slowly increased to 100 bars.(32) The materials were allowed to set for the time specified by manufactures for commercial materials and 10 minutes forexperimental materials. The samples were removed carefully from the mould and inspected to exclude any specimens with a visible void or defect. All specimens were stored in plastic containers at $23 \pm 1{ }^{\circ} \mathrm{C}$ until further use.Samples were handled with forceps throughout the experiment to avoid the finger contact and any contamination of the testing surfaces.

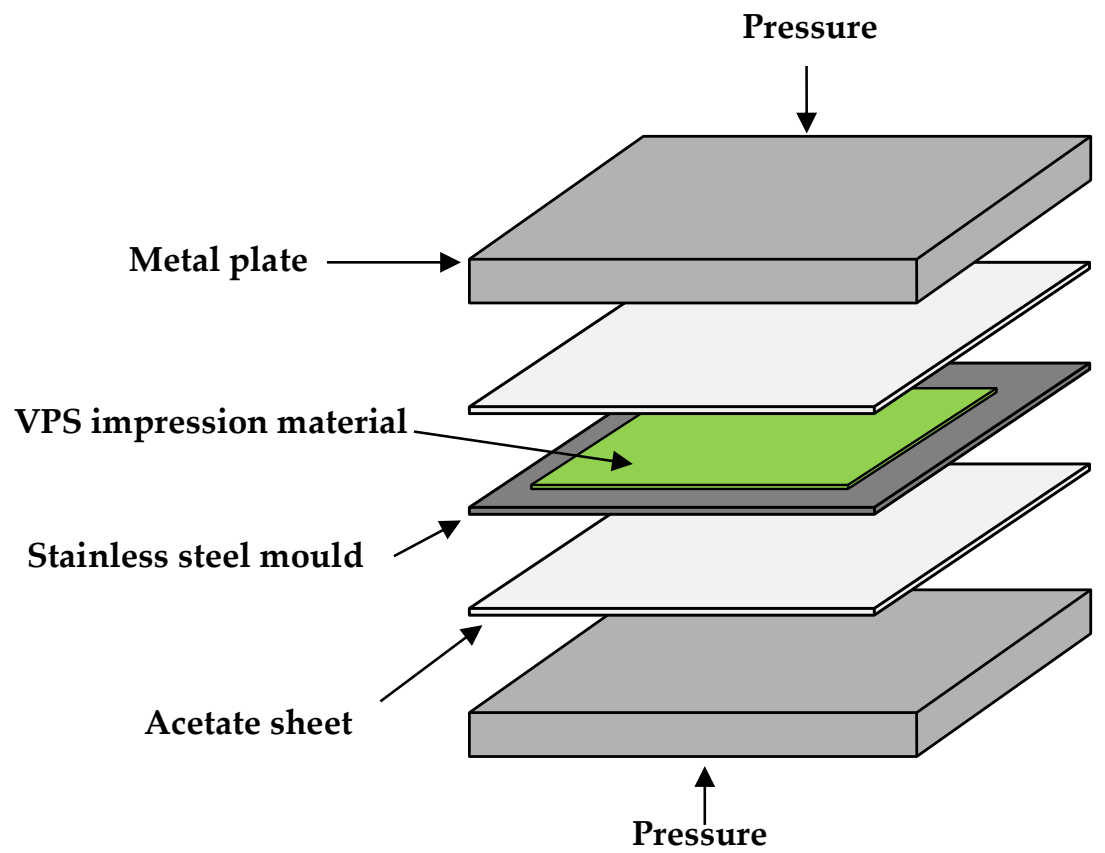

Fig. 1. Schematic presentationof components used for sample preparation for contact angle measurements

\subsection{Contact angle measurement}


A Drop Shape Analyser (Model DSA-100; KRÜSS GmbH, Germany) was used for the contact angle measurements. This equipment uses LED illumination and a precise lens. The lens's large zoom range enables reliable data even in case of small drops with optimum width.A number of researchers $(20,21,33-36)$ have used the DSA100 device to measure the contact angles and complementary software (DSA1) to analyse the data (Figure 2).

For this purpose, each sample was carefully placed on the sample table and the video recorder was started. A droplet of deionised distilled water $(\sim 1.5 \mu \mathrm{L})$ was placed on the impression material surface using a Gastight \#1001 syringe (Hamilton Bonaduz AG, Switzerland - accuracy to $0.01 \mathrm{ml}$ ). The contact angles were measured and corresponding images were recorded at time intervals of 10, 30, 60 and 120 seconds (19-21,37). Before every session of measurements, the equipment was calibrated using the Young-Laplace principle (38). Briefly, glass panels with images of standard drop shapes with known contact angles $\left(30^{\circ} \pm 0.09^{\circ}, 60^{\circ} \pm 0.07^{\circ}\right.$ and $\left.120^{\circ} \pm 0.04^{\circ}\right)$ were used as standards. The DSA1 software program (V. 1.92-06; TrackerMan option, KRÜSS GmbH, Germany) was used for calibration measurements. The measuring parameters were entered in the TrackerMan of DSA1 program and the reliability of the measuring system was checked by comparing the measured values with the standards.

(a)

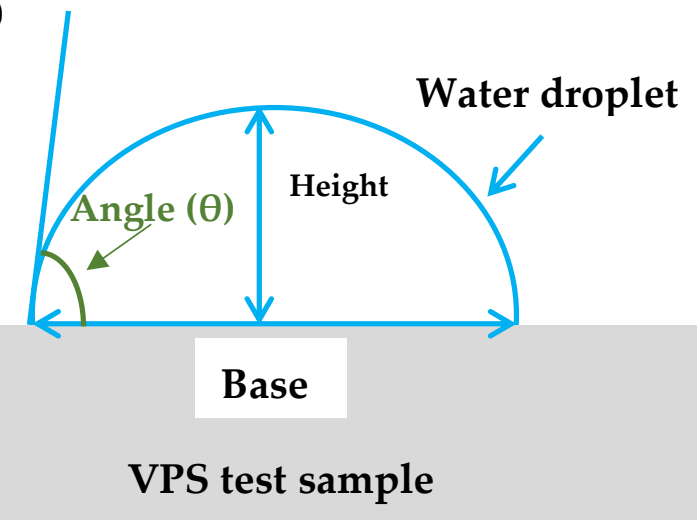

(b)

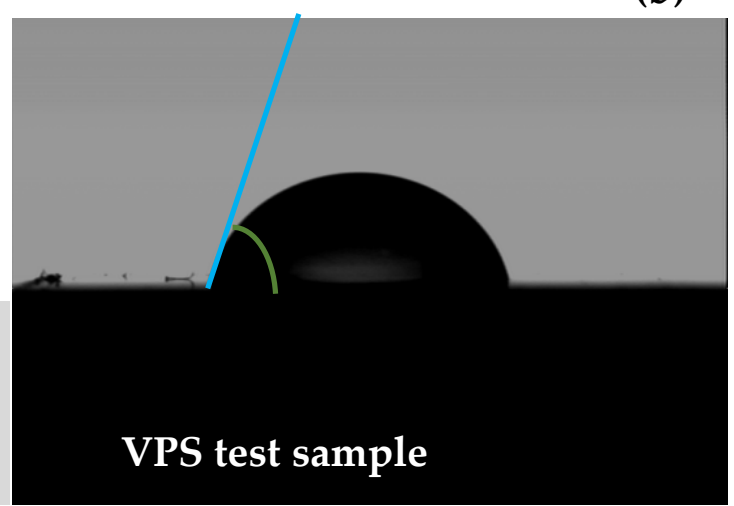

Fig.2. Contact angle measurement process; (a) Schematic presentation(b) Representative images of contact angle measurement of VPS impression materials obtained from DSA1 software program. 
The images were further processed by the software (DSA1) was used to process images and analyse the contact angles variations at 10, 30, 60 and 120 seconds. An average of 10 $(\mathrm{n}=10)$ measurements (Mean \pm standard errors)were calculatedfor each impression materials [three commercial materials and Exp-I, II, (no surfactant) Exp-III, IV, V (containing 2\%, $2.5 \%$ and 3\% Rhodasurf CET-2 respectively), and Exp-VI (containing 2\% Rhodasurf CET5)] immediately after setting, after immersion in deionised water and disinfectant (1\% $\mathrm{NaOCl})$.

\subsection{Statistical analysis}

Data were analysed using the SPSS statistical 22 software (IBM, USA). To analyse the significant differences between the means of the groups of the materials, one way analysis of variance (ANOVA) and Tukey's Honest Significant Difference (HSD) were applied. Further analyses of the data were performed using a post hoc test (Tukey's HSD test) to determine which means of the groups were significantly different from each other. The differences were considered significant at the $\mathrm{p}<0.05$ level.

\section{RESULTS}

\subsection{Contact angles upon setting}


The contact angles of commercially available and experimental materials were calculated immediately after the completion of setting reaction (Figure 3 ).

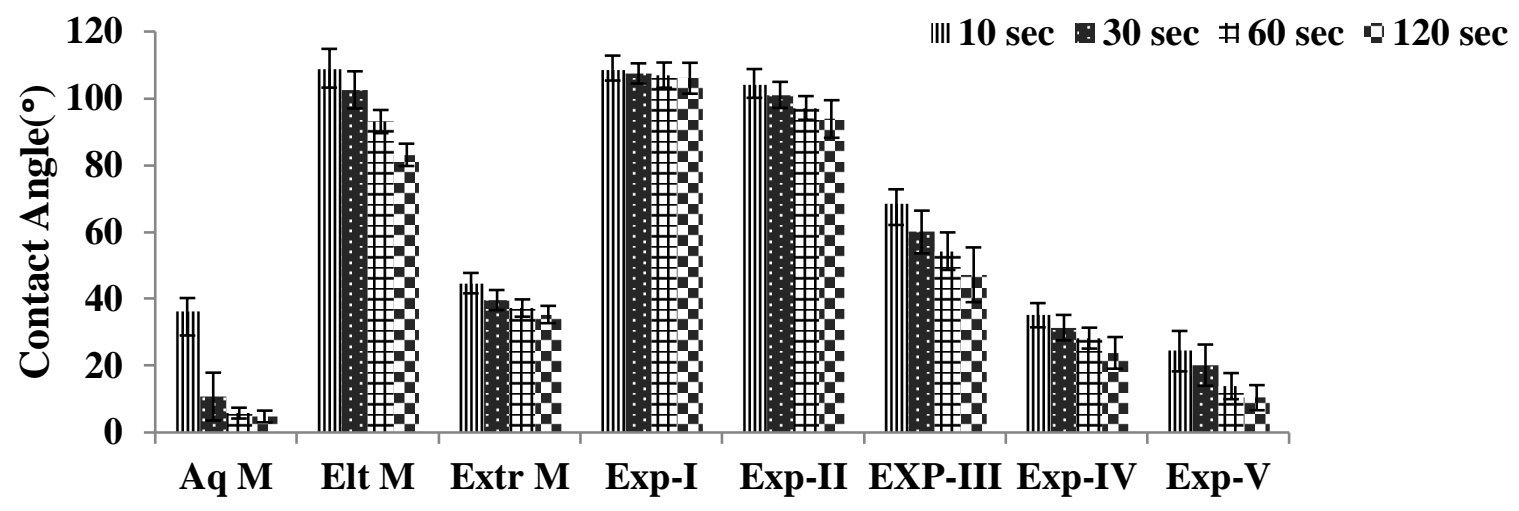

Fig. 3. Contact angles of various commercial and experimental VPS materialsmeasured immediately after setting $(n=10)$.

Among the commercial materials, the largest contact angle (at $10 \mathrm{sec}$ ) was recorded for Elt $M(108.78 \pm 6.04)$ that was significantly larger $(p<0.05)$ than Aq M $(36.19 \pm 4.05)$ and Extr M (44.65 \pm 3.11$)$. The contact angles (at $10 \mathrm{sec})$ for experimental materials Exp-I $(108.35 \pm 4.45)$, Exp-II (104.08 \pm 4.70$)$ closely matched to Elt M (108.78 \pm 6.04$)$ however were significantly larger $(\mathrm{p}<0.05)$ than other experimental materials, Exp-III $(68.50 \pm 4.29)$, Exp-IV

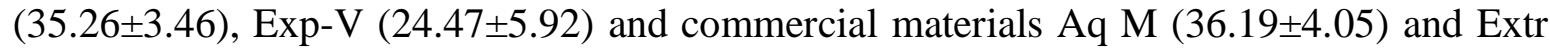
$M(44.65 \pm 3.11)$. The variation in the contact angles of all materials were observed for two minutes; there was a consistent and significant decrease in contact angles within two minutes (Figure 3). There were no significance differences in contact angles between consecutive measurements. The only exception was Aq M where the contact angle measured at 10 sec $(36.19 \pm 4.05)$ was significantly $(\mathrm{p}<0.05)$ lowered at $30 \mathrm{sec}(10.75 \pm 7.15)$.

\subsection{Effects of water immersion on Contact angles}

Upon immersion in the deionised water for one week, all materials revealed a significant $(\mathrm{p}<0.05)$ increase in the contact angles compared to corresponding values measured 
immediately after setting. The largest contact angle was recorded for Elt M (115.28 44.19$)$ in commercial materials and Exp-I (120.13 \pm 4.94$)$ among the experimental materials. However, both materials did not reveal significant differences compared to contact angles recorded for

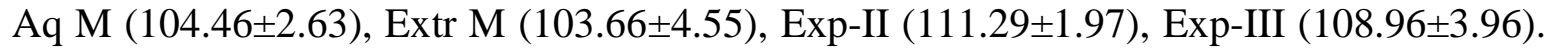
In contrast, the contact angles for Exp-IV (80.71 \pm 5.99$)$ and Exp-V (77-03 \pm 6.00$)$ were significantly lower compared to all other materials suggesting their more hydrophilicity while immersion in deionised water (Figure 4).

In terms of measurement time (10-120 seconds), there was a generalised trend of lowering contacts angles gradually with time among consecutive measurements; however, significant differences $(p<0.05)$ were observed in case of Aq M, Extr M, Exp-IV and Exp-V materials (Figure 4).

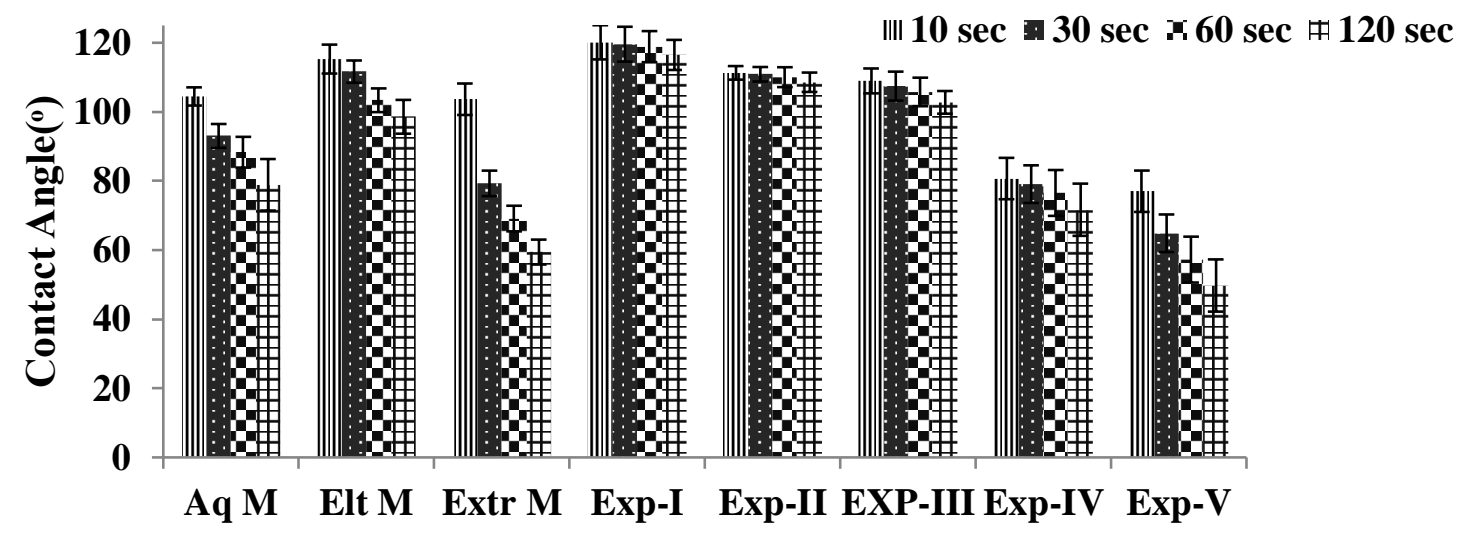

Fig. 4.Contact angles of various commercial and experimental VPS materialsmeasured after 1 weekimmersion in deionized water $(n=10)$.

The contact angle measured at $120 \mathrm{sec}$ showed significantly larger values $(\mathrm{p}<0.05)$ for

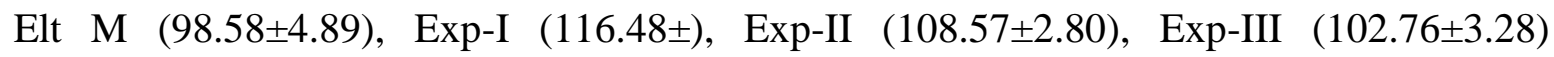

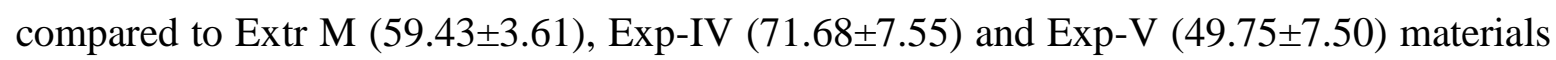
(Figure 4).

\subsection{Effects of disinfectant $(1 \% \mathrm{NaOCl})$ immersion on contact angles}


The contact angles of all materials following immersion in disinfectant $(1 \% \mathrm{NaOCl})$ were not significantly different compared to contact angles of materials immersed in the deionised water (Figure 4). The only exception was Exp-V that had contact angle of $77.03 \pm 6.00$ while immersed in the deionised water and significantly reduced $(\mathrm{p}<0.05)$ while immersed in the $1 \% \mathrm{NaOCl}$ disinfectant $(52.37 \pm 5.63)$. In contrast, disinfectant immersion

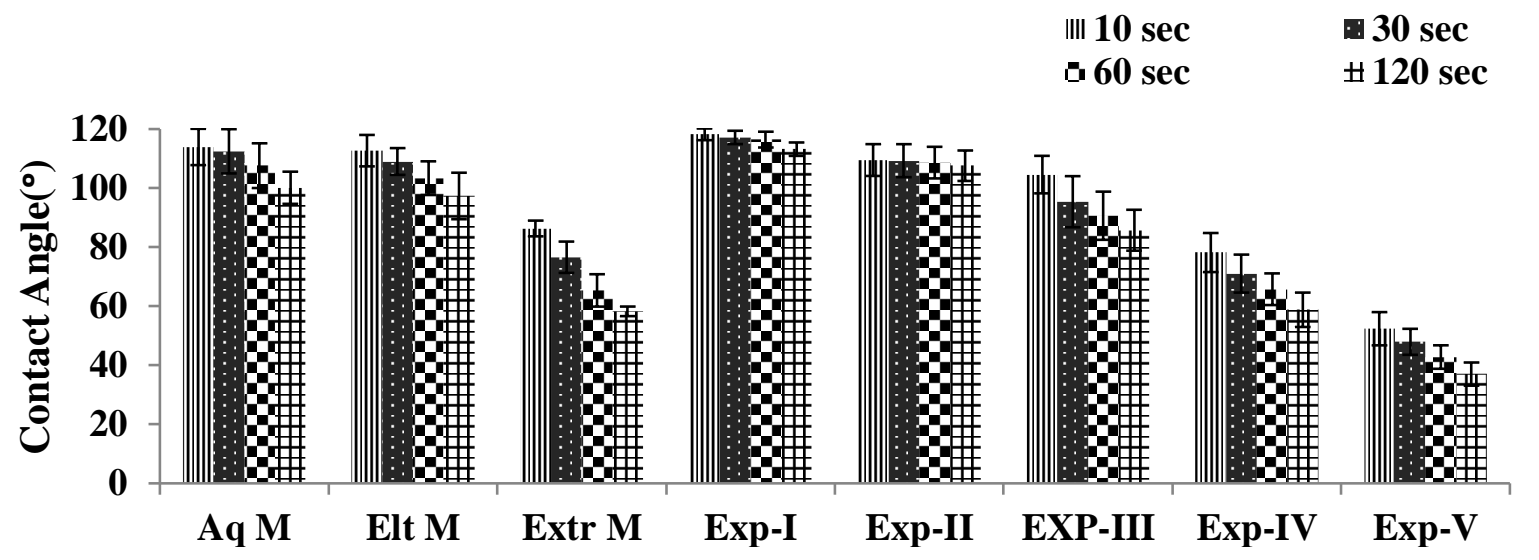

Fig. 5. Contact angles of various commercial and experimental VPS materialsmeasured after 1 week disinfection in $1 \% \mathrm{NaOCl}(\mathrm{n}=10)$.

significantly increased $(\mathrm{p}<0.05)$ the contact angles for all materials compared to contact angles immediately after setting (Figure 3 and 5). Although the contact angles for each materials were gradually reduced at each time interval; Aq M, Elt M, Exp-I, Exp-II and ExpIII showed no significant differences $(\mathrm{p}>0.05)$ between contact angles measured at two consecutive time intervals. In case of Extr M, Exp-IV and Exp-V, there was a significant $(\mathrm{p}<0.05)$ reduction in the contact angle at each time interval (Figure 5).

\subsection{Effect of Rhodasurf CET-2 and CET-5 on contact angles}


In order to study the effects of surfactants molecular weight on contact angles, Exp-III (contained 2\% Rhodasurf CET-2) and Exp-VI (contained 2\% Rhodasurf CET-5) were compared immediately after setting and disinfectant treatment. Contact angles measured

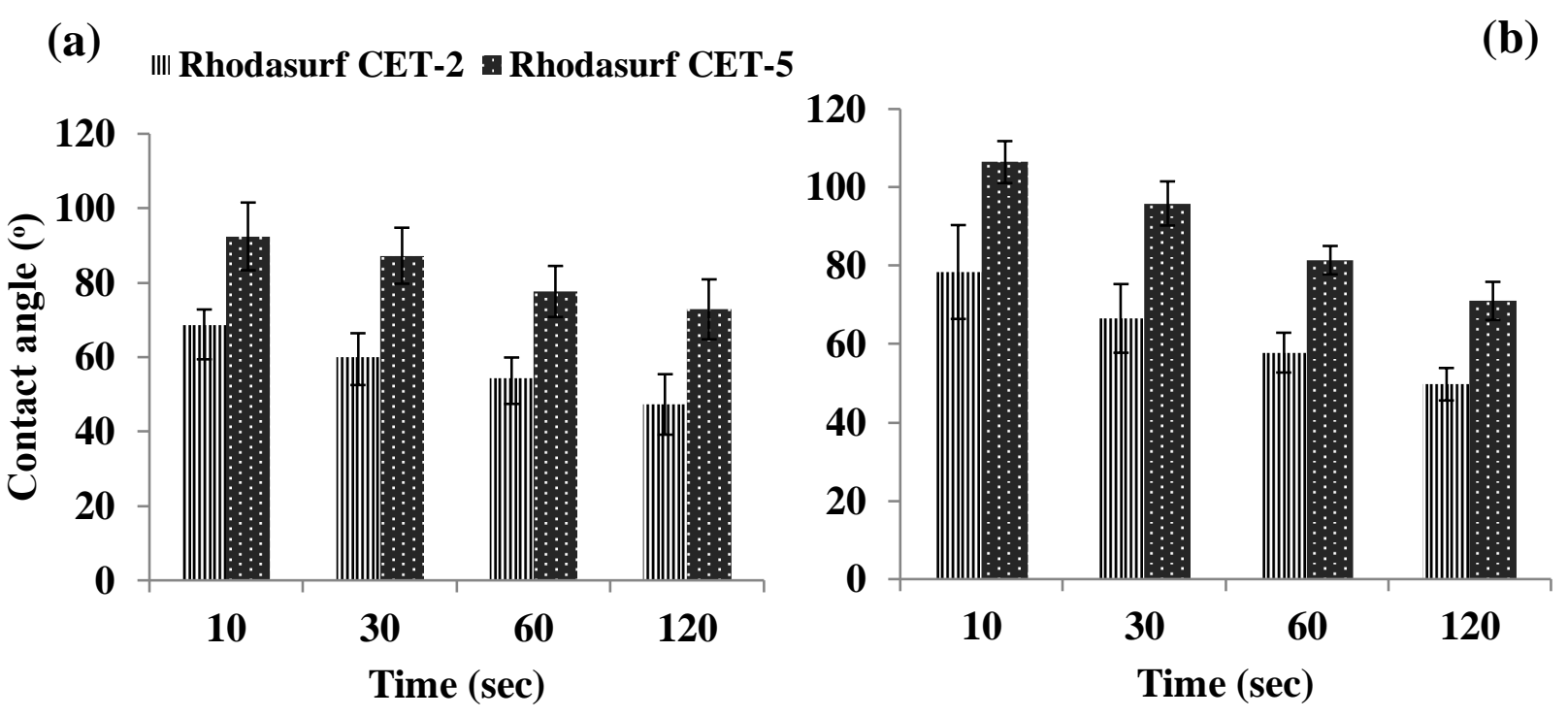

Fig. 6.Contact angles of experimental VPS materialsExp-III and Exp-VI (containing Rhodasurf CET-2 and Rhodasurf CET-5 respectively) (n=10); (a) Immediately after setting, (b) After 1week disinfection in $1 \% \mathrm{NaOCl}$

immediately after settings showed that the contact angle (measured at $10 \mathrm{sec}$ ) of Exp-III $(68.5 \pm 4.3)$ was significantly $(\mathrm{p}<0.05)$ smaller than Exp-VI $(92.4 \pm 9.1)$. The differencein contact angles of Exp-III and Exp-VI remained significant at each time interval. There was gradual and insignificant $(\mathrm{p}>0.05)$ reduction in contact angles of both materials at each time interval (Figure 6A).

The treatment with disinfectant $(1 \% \mathrm{NaOCl})$ resulted in an increase in the contact angles of both material regardless of time interval. The measurement at $10 \mathrm{sec}$,Exp-III contact angle was increased from $68.5 \pm 4.3$ (setting) to $78.4 \pm 12.0$ (disinfected) that was not significant. Similar trendswere observed for measurement made at 30,60 and 120 seconds. In contrast, the contact angle (measured at $10 \mathrm{sec})$ for Exp-VI was increased significantly $(\mathrm{p}<0.05)$ from $92.4 \pm 9.1$ (setting) to $106.5 \pm 5.4$ (disinfected). For any time interval, the contact angle for ExpVI remain significantly larger compared to corresponding contact angle of Exp-III. The 


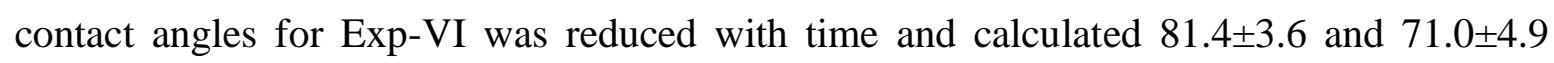
respectively for $60 \mathrm{sec}$ and $120 \mathrm{sec}$ (Figure 6B).

\section{DISCUSSION}

The current study explored theeffects of adding hydrophilic agents (Rhodasurf CET-2 and CET-5)to the vinyl polysiloxane (VPS) impression materials. The surfactant's molecular weight and wetting properties (such as hydrophilicity, contact angles) were considered upon immersion in water and disinfectant medium. The addition of surfactant (Rhodasurf CET-2) reduced the contact angles vinyl polysiloxane impression materials. Furthermore, the concentration and smaller molecular weight of the surfactants affected the contact angles. The wettability of all materials was affected upon immersion in water and disinfectant solutions. Disinfection of impression materials after removing from the mouth is paramount to prevent cross-infection. However, disinfecting solutions may adversely affect the hydrophilicity of VPS impression materials leading to create issues of compatibility of impressions with gypsum slurries. In previous studies researchers have investigated the hydrophilicity and dimensional stability of commercial impression materials in different disinfecting solutions(30,31, 39). However, there are no report of developingtailored formulations. It is quite difficult to understand and explain the behaviour of a material without knowing its composition. A number of researchers have developed their own compositions (20,21)however the material's response to disinfectants was not evaluated. Hence, in this contribution all materials were disinfected prior to evaluating their performance.

In order to address the above issues, and to study the effects of, for example, incorporating hydrophilic agents, the exact composition of components within VPS was required. Therefore experimental (Exp) VPS impression materials were developed, with known compositions, so that the results (e.g. hydrophilicity) could be argued criticallybefore and after long term (1 week) immersion in disinfecting solution $(1 \% \mathrm{NaOCl})$. Furthermore, 
the hydrophilicity of the latter could then be compared with commercial VPS impression materials. The contact angles for the hydrophilic Exp VPS (Exp-III, IV and V), showed significantly lower values compared to their corresponding controls (Exp-I and Exp-II) and these were concentration dependent. The contact angle results for Aq $\mathrm{M}$ after immersion in $1 \% \mathrm{NaOCl}$ for 1 week were surprising; the contact angles had increased significantly, by $\sim 90^{\circ}$ after disinfection for 1 week compared to immediately after setting (Figure4 and 5). Furthermore, at one week CAs were above $100^{\circ}$ and hence, at this stage Aq M can no longer be classified as a hydrophilic impression material (Figure5). The CAs of Exp-IV and Exp-V (Figure4 and 5) were also affected by DI but to a lesser extent. A similar trend of increasing contact angle was observed in distilled water (Figure6). These results suggest that the surfactant is leaching out into the disinfecting solution with time. Hence, leaving the materials in disinfecting solutions for longer periods is likely to compromise the wettability of hydrophilic VPS impression materials and should be avoided.The contact angle results obtained for the Exp formulations suggest that Rhodasurf CET-2 is a better surfactant compared to others, for example nonylphenoxy poly(ethyleneoxy), because it lowers the contact angle hence making the materials hydrophilic, secondly it does not leaches rapidly during disinfection. To explain this it is important to look at the chemical structure of the non-ionic surfactant, Rhodasurf CET-2, (ethoxylatedcetyl-oleyl alcohol), which is a mixture 
of ethoxylatedcetyl and ethoxylatedoleyl alcohols (as shown in Figure 7A and 7Brespectively).

(a)

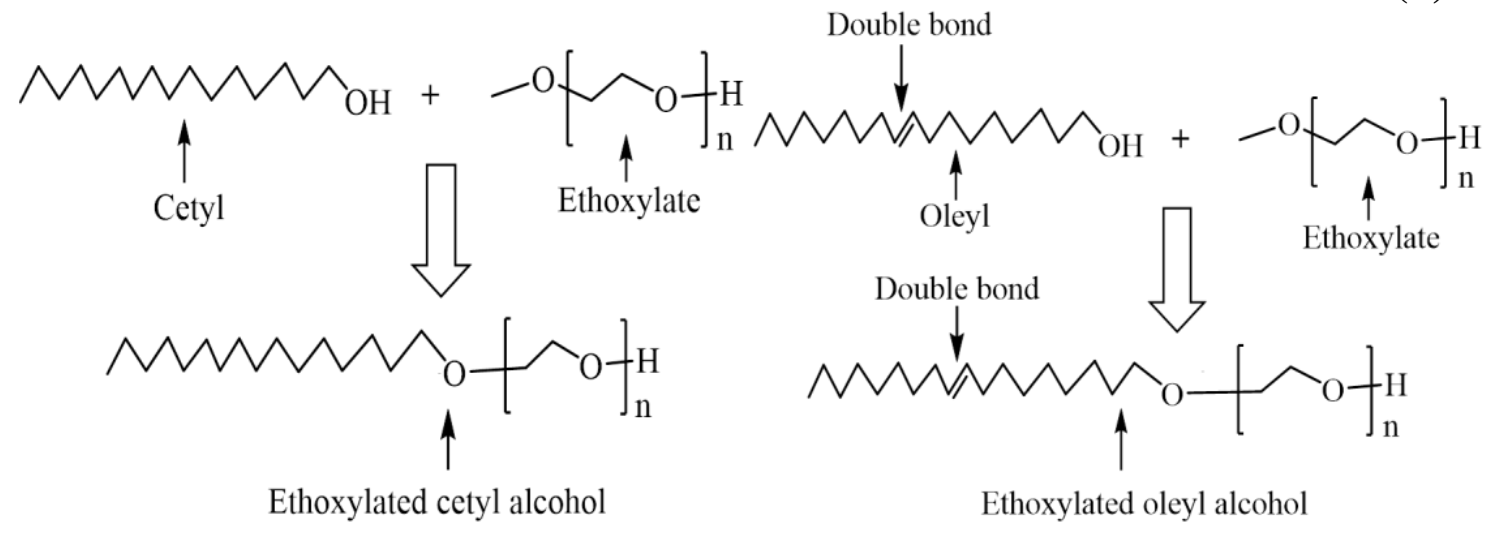

Fig. 7. Chemical structure of (a) ethoxylatedcetyl alcohol; a part of ethoxylatedcetyl-oleyl alcohol; (b)ethoxvlatedolevl alcohol that is other part of ethoxvlatedcetvl-olevl alcohol.

It is interesting to note that ethoxylatedoleyl alcohol (Rhodasurf CET-2) contains a double bond in its chemical structure (Figure7). Could it be possible that some components in the Exp compositions could activate this double bond? If the answer to this question is yes, then it is assumed that this double bond may also polymerise during setting and may not leach out during disinfection and thus contributes to improve wettability. If the answer to this question is no, then there must be strong intermolecular (Van der Waals) forces or molecular entanglement which limit the leaching of the surfactant. Clearly, there is need of further work in this area because there is no sufficient literature available on the use of such surfactants in impression materials. Therefore, it is difficult to compare our findings with other studies.

It is not known what surfactant Elt $M$ and Extr M contain, whereas a US patent (40) on Aq M reports that Igepal CO-530 (nonylphenoxy poly [ethyleneoxy] ethanol), is incorporated as a non-ionic surfactant, which is a surface acting agent that is soluble in water. It is more widely used as a detergent. The hydrophilic group of the non-ionic surfactant is a polymerised alkene oxide water soluble polyether. It is apparent from its chemical structure (Figure7) that it does not contain a double bond and so it is assumed that it is not chemically bonded to the 
silicone polymer matrix. Therefore, due to the latter, and the surfactant's solubility, resulted in it leaching, thus causing an increase in contact angle during immersion in solutions. From the limited information obtained from the Materials Safety Data Sheets (MSDS) on Rhodasurf CET-2 and Igepal CO-530, it seems that the latter is more soluble in water and has a lower $\mathrm{Mw}$ than the former. These factors could also contribute the Igepal CO-530 leaching with ease after disinfection at different time points (minimum disinfection time $=30$ minutes). Lepe et al (30) have suggested that the intrinsic surfactants (e.g. Igepal) added to the hydrophilic VPS impression materials may be washed out during disinfection.

It is evident from the previous literature that the molecular weight of the surfactant affects the contact angle of the material $(20,21)$. In the current study this is confirmed where Exp-VI with the surfactant Rhodasurf CET-5 (2\% by weight), which has a larger molecular size, gave higher CAs compared with formulation Exp-III containing the lower molecular size, Rhodasurf CET-2 (2\% by weight). It is assumed here that Rhodasurf CET-2, with its smaller molecular size, can diffuse to the surface of the material with ease, compared to the larger molecular weight surfactant, thus making the surface more wettable. Similar findings were reported by researchers $(20,21)$ who also used different molecular weight surfactants. Please delete repetition from above.Our results showed that the contact angles were higher for all commercial and Exp materials at 10 seconds compared to contact angles at two minutes. These findings are suggestive that the surfactant travels to the surface of the material with time, thus reducing contact angles. This theory is also supported by a study conducted by Grundke et al (9).

To verify their efficacy, the novel experimental materials were compared with the commercial VPS impression materials. This landmark study can prove to be turning point in the way clinicians ought to handle VPS impression materials. The results can be used to formulate guidelines for clinicians in cases where an impression remains immersed in disinfecting solution for longer periods of time (from days to a week). 


\section{CONCLUSION}

The experimental vinyl polysiloxane materials containing the surfactant (Rhodasurf CET-2) showed lower contact angles compared to controls (Exp-I and Exp-II) and commercial vinyl polysiloxane impression materials. The decreasein contact angles was dependent on the concentration of surfactant (Rhodasurf CET-2) in the materials. In addition, the smaller molecular weight surfactant (Rhodasurf CET-2) resulted in lower contact angles compared to the largermolecular weight surfactant (Rhodasurf CET-5). The wettability of all materials was affected upon immersion in deionised water and disinfectant solutions. This factor is of clinical significance and must be considered while washing and disinfection the set impressions prior to making dental casts.

Conflicts of Interest: The authors declare no conflict of interest.

\section{References and Notes}

1. S. Deb; Polymers in dentistry; Proc Inst Mech Eng Part H J Eng Med 212, 453 (1998).

2. J. De Clerck; Microwave polymerization of acrylic resins used in dental prostheses; $J$ Prosthet Dent 57, 650 (1987).

3. M. S. Zafar, and N. Ahmed; Nanoindentation and surface roughness profilometry of poly methyl methacrylate denture base materials; Technology and Health Care 22, 573 (2014).

4. M. A. Abuzar, S. Bellur, N. Duong, B. B. Kim, P. Lu, N. Palfreyman, D. Surendran, and V. T. Tran; Evaluating surface roughness of a polyamide denture base material in comparison with poly (methyl methacrylate). J Oral Sci 52,(2010).

5. P. Vallittu; Some aspects of the tensile strength of unidirectional glass fibre-polymethyl methacrylate composite used in dentures; J Oral Rehabil 25, 100 (1998).

6. S. Najeeb, M. S. Zafar, Z. Khurshid, and F. Siddiqui; Applications of polyetheretherketone (PEEK) in oral implantology and prosthodontics; Journal of prosthodontic research 60, 12 (2016).

7. A. Schwitalla, and W. Müller; PEEK dental implants: a review of the literature; $J$ Oral Implantol 39, 743 (2013).

8. S. Najeeb, Z. Khurshid, S. Zohaib, and M. S. Zafar; Bioactivity and osseointegration of PEEK are inferior to those of titanium-A systematic review. J Oral Implantol 42, 512 (2016). 
9. K. Grundke, S. Michel, G. Knispel, and A. Grundler; Wettability of silicone and polyether impression materials: Characterization by surface tension and contact angle measurements; Colloids Surf Physicochem Eng Aspects 317, 598 (2008).

10. A. J. Raigrodski, S. Dogan, L. A. Mancl, and H. Heindl; A clinical comparison of two vinyl polysiloxane impression materials using the one-step technique; J Prosthet Dent 102, 179 (2009).

11. S. Levartovsky, M. Folkman, E. Alter, and R. Pilo; Elastomeric impression materials; Refuat Hapeh Vehashinayim (1993) 28, 54 (2011).

12. N. C. Lawson, J. O. Burgess, and M. Litaker; Tear strength of five elastomeric impression materials at two setting times and two tearing rates; Journal of Esthetic and Restorative Dentistry 20, 186 (2008).

13. T. A. Hamalian, E. Nasr, and J. J. Chidiac; Impression materials in fixed prosthodontics: influence of choice on clinical procedure; Journal of Prosthodontics 20, 153 (2011).

14. S. Chen, W. Liang, and F. Chen; Factors affecting the accuracy of elastometric impression materials; J Dent 32, 603 (2004).

15. R. S. Kess, E. C. Combe, B. S. Sparks, and U. of Minnesota; Effect of surface treatments on the wettability of vinyl polysiloxane impression materials; J Prosthet Dent 84, 98 (2000).

16. T. S. Menees, R. Radhakrishnan, L. C. Ramp, J. O. Burgess, and N. C. Lawson; Contact angle of unset elastomeric impression materials; J Prosthet Dent 114, 536 (2015).

17. W. W. Chee, and T. E. Donovan; Polyvinyl siloxane impression materials: a review of properties and techniques; J Prosthet Dent 68, 728 (1992).

18. C. S. Petrie, M. P. Walker, A. M. O'Mahony, and P. Spencer; Dimensional accuracy and surface detail reproduction of two hydrophilic vinyl polysiloxane impression materials tested under dry, moist, and wet conditions; J Prosthet Dent 90, 365 (2003).

19. D. Pratten, and R. Craig; Wettability of a hydrophilic addition silicone impression material; J Prosthet Dent 61, 197 (1989).

20. Y. Oh, D. Lee, S. Hwang, K. Kim, and K. Kim; Effect of non-ionic surfactants on surface properties of hydrophilic polyvinyl siloxane impression materials; Colloids Surf Physicochem Eng Aspects 229, 9 (2003).

21. D. Lee, Y. Oh, K. Chung, K. Kim, and K. Kim; Mechanism study on surface activation of surfactant-modified polyvinyl siloxane impression materials; J Appl Polym Sci 92, 2395 (2004).

22. S. Minagi, K. Fukushima, N. Maeda, K. Satomi, S. Ohkawa, Y. Akagawa, Y. Miyake, H. Suginaka, and H. Tsuru; Disinfection method for impression materials: freedom from fear of hepatitis B and acquired immunodeficiency syndrome; J Prosthet Dent 56, 451 (1986).

23. B. K. Norling, and M. H. Reisbick; The effect of nonionic surfactants on bubble entrapment in elastomeric impression materials; J Prosthet Dent 42, 342 (1979).

24. Z. Iqbal, and M. S. Zafar; Role of antifungal medicaments added to tissue conditioners: A systematic review; Journal of Prosthodontic Research 60, 231 (2016).

25. D. Hosseinpour, and J. C. Berg; The dynamic interaction of water with four dental impression materials during cure; Journal of Prosthodontics 18, 292 (2009). 
26. N. Martin, M. Martin, and N. Jedynakiewicz; The dimensional stability of dental impression materials following immersion in disinfecting solutions; Dental materials 23, 760 (2007).

27. T. M. Fabian, and S. E. Walker; Stability of sodium hypochlorite solutions; Am J Hosp Pharm 39, 1016 (1982).

28. ADA COUNCIL ON SCIENTIFIC AFFAIRS, and PRACTICE, ADA COUNCIL ON DENTAL; Infection control recommendations for the dental office and the dental laboratory; J Am Dent Assoc 127, 672 (1996).

29. S. Ahmad, C. Tredwin, M. Nesbit, and D. Moles; Effect of immersion disinfection with Perform-ID on alginate, an alginate alternative, an addition-cured silicone and resultant type III gypsum casts; Br Dent J 202, E1 (2007).

30. X. Lepe, G. H. Johnson, J. C. Berg, T. C. Aw, and G. S. Stroh; Wettability, imbibition, and mass change of disinfected low-viscosity impression materials; J Prosthet Dent 88, 268 (2002).

31. B. R. Lang, X. Lepe, G. H. Johnson, and J. C. Berg; Surface characteristics of polyether and addition silicone impression materials after long-term disinfection; J Prosthet Dent 74, 181 (1995).

32. I. M. Meththananda, S. Parker, M. P. Patel, and M. Braden; The relationship between Shore hardness of elastomeric dental materials and Young's modulus; Dental materials 25, 956 (2009).

33. F. Rupp, D. Axmann, A. Jacobi, M. Groten, and J. Geis-Gerstorfer; Hydrophilicity of elastomeric non-aqueous impression materials during setting; Dental Materials 21, 94 (2005).

34. J. Seo, and L. P. Lee; Effects on wettability by surfactant accumulation/depletion in bulk polydimethylsiloxane (PDMS); Sensors Actuators B: Chem 119, 192 (2006).

35. G. Kugel, T. Klettke, J. A. Goldberg, J. Benchimol, R. D. Perry, and S. Sharma; Investigation of a new approach to measuring contact angles for hydrophilic impression materials; Journal of Prosthodontics 16, 84 (2007).

36. M. Balkenhol, S. Haunschild, G. Lochnit, and B. Wöstmann; Surfactant release from hydrophilized vinylpolysiloxanes; J Dent Res 88, 668 (2009).

37. K. X. Michalakis, A. Bakopoulou, H. Hirayama, D. P. Garefis, and P. D. Garefis; Preand Post-set Hydrophilicity of Elastomeric Impression Materials; Journal of Prosthodontics 16, 238 (2007).

38. J. Pellicer, V. Garcia-Morales, and M. Hernández; On the demonstration of the YoungLaplace equation in introductory physics courses; Physics Education 35, 126 (2000).

39. M. P. Walker, M. Rondeau, C. Petrie, A. Tasca, and K. Williams; Surface quality and long-term dimensional stability of current elastomeric impression materials after disinfection; Journal of Prosthodontics 16, 343 (2007).

40. R. Hare; Polyorganosiloxane dental impression materials with improved wetting and stability; U.S. Patent Application No. 11/650,770 (2007). 